\title{
Fluorescence imagine of retinal microglial cells in rodent and primate under degenerative and regenerative conditions throughout aging
}

\author{
Hanhan Liu', Fabian Anders ${ }^{2}$, Solon Thanos ${ }^{2}$, Karl Mercieca ${ }^{3}$ and Verena Prokosch ${ }^{1 *}$ \\ ${ }^{1}$ University of Cologne, Faculty of Medicine and University Hospital Cologne, Department of Ophthalmology, 50937, Cologne, Germany \\ ${ }^{2}$ Department of Ophthalmology, University Medical Center of the Johannes Gutenberg University Mainz, 55131, Mainz, Germany \\ ${ }^{3}$ Department of Experimental Ophthalmology, School of Medicine, University of Münster, 48149, Münster, Germany \\ ${ }^{4}$ Department of Ophthalmology, University Medical Center of Bonn, 53127 Bonn, Germany
}

\begin{abstract}
Purpose: Microglia play a pivotal role in neurodegeneration, -regeneration and aging within the central nervous system (CNS). Purpose of this study was to analyze and compare the role of microglia in regeneration, degeneration, changes throughout ageing and within different species.

Methods: Elevated intraocular pressure (IOP) was induced in vivo in female Sprague-Dawley (SD) rats by cauterization of three episcleral veins (n=5). Ex vivo, retinal explants $(\mathrm{n}=8)$ of different aged animals ( 3 months and 24 months) were harvested and cultured with or without elevated pressure $(40 \mathrm{mmHg})$ for $48 \mathrm{hours.} \mathrm{In}$ addition, retinal explants from rats after optic nerve crush $(n=5)$ and monkeys (Callithrix jacchus, $n=4)$ were cultured in vitro under conditions allowing spontaneous regeneration of axons. Immunohistochemical staining against Iba-1 was employed to grade and quantify microglia cells in retinal explants. Untreated samples served as the controls, respectively. Statistical analysis was performed to compare groups.

Results: Under degenerative conditions both in vivo and ex-vivo a significant microglial activation is shown ( $<<0.001$ ), while absolute number of microglia remained stable. In terms of aging, old animals possessed significantly more microglia, and also a significant rise of activated microglia in the older animals ( $<<0.0001)$. Under regenerative conditions significantly less activation was visible. However, in primates, microglia showed opposite activation patterns under regenerative conditions.

Conclusions: Both degenerative and regenerative conditions resulted in microglial activation. Regenerative conditions showed only moderate activation. There is an age-related decrease in microglial function. Last but not least, conclusions drawn from rodents and cell studies can not necessarily be translated to mammalian microglia.
\end{abstract}

\section{Introduction}

Microglia is the most common innate immune cell resident in the CNS. $10-15 \%$ of all glial cells is microglia and they are often referred to as the tissue-resident macrophages of the CNS [1,2]. It is very well known that microglia play a tremendous role in CNS diseases, as microglia process remarkable plasticity and capability of responding swiftly to damage or infection, where they undergo functional and morphological changes as a reaction to neuronal damage and external stimulus [3-40]. Those changes are connected with a visible cellular transformation from a resting phenotype to an activated status to perform inflammatory functions[19,41]. Resting microglia in the CNS have a small cell body and fine cellular processes, and are highly ramified-a morphology that distinguishes them from macrophages and dendritic cells [15]. The morphology of activated microglia includes a retraction of processes, enlargement of the soma, and increased expression of myeloid cell markers [35]. Resting microglia are not truly "resting", these cells participate in CNS development, homeostasis, and nearly all CNS disturbances. As part of their homeostatic functions, microglial cell bodies remain stationary, but their processes continuously scan the surrounding extracellular space and communicate directly with neurons, astrocytes, and blood vessels [29]. In response to injury and degeneration, microglia become activated, change their morphology, proliferate, migrate to the damage sites, modify the expression of enzymes and receptors $[16,27,34]$.

One of the main functions of activated microglia is to regulate CNS homeostasis and execute appropriate responses, such as releasing a variety of cytokines, including inflammatory factors, such as NO, tumor necrosis factor (TNF- $\alpha$ ), interleukin (IL-6) among others [26]. Neuroinflammation is a response fundamentally initiated to protect CNS. But uncontrolled microglia activation can lead to excessive inflammation, furthermore put neuronal survival in danger. Especially when neurodegenerative CNS disorders, including multiple sclerosis (MS), Alzheimer's disease (AD), Parkinson's disease (PD), Huntington's disease (HD), are associated with neuroinflammation[6].[25]. While CNS is affected by neurodegenerative diseases like Alzheimer's, the

${ }^{\star}$ Correspondence to: Verena Prokosch, University of Cologne, Faculty of Medicine and University Hospital Cologne, Department of Ophthalmology, 50937, Cologne, Germany, E-mail: verena.prokosch-willing@uk-koeln.de

Key words: glaucoma, retinal biomarkers, microglia, degeneration, regeneration

Received: September 10, 2020; Accepted: February 15, 2021; Published: February 23, 2021 
retina is affected by glaucoma, both of them being recognized as agerelated diseases [33].

Recruitment of microglial cells seems to have a direct influence on neuronal cell death in glaucoma $[4,20]$, causing an apoptotic stimulus by yet not fully understood cellular mechanisms [23] and possible secretion of highly functional, inflammatory mediators into the surrounding cell network $[9,10]$.

While the role of microglia in a degeneration, like glaucoma, was investigated deeply in recent years, their differentiated function in neuronal regeneration is rather unknown until today. Furthermore, age-related changes need further investigation and it is completely unclear whether research done in rodents applies to primates.

In this study, quantification and qualification of microglia were performed in experimental glaucoma models of rats in vivo and ex vivo as well as in retinal flat-mounts under regenerative conditions. The role of microglia in different pathophysiological conditions of glaucoma is unknown and might vary according to age and species. Purpose of this study was to give for the first time a comprehensive overview of 1. Age- dependent differences in microglial responses; 2 . its role in degeneration and regeneration and; 3 . differences between rodents and primates.

\section{Methods}

\section{Animal statement}

All experiments were conducted in accordance with the Association of Research in Vision and Ophthalmology Statement for the Use of Animals in Ophthalmic and Vision Research. The study was approved by the Committee of Animal Research Rhineland-Palatinate (permission-no: 14-1-085). Retinae of primates (Callithrix jacchus, 8 -year-old, $n=4$ ) were obtained from eyes provided by the Institute of Regenerative Medicine, University of Münster, Germany (permissionno: 8.87-50.10.46.09.018 and 39.32.7.1/39.32.7.2.1). All used animals, for experimental glaucoma in vivo as well as ex vivo experiments, were female Sprague Dawley rats exclusively. Ex-vivo samples of Callithrix jacchus aged 3 years $(n=4)$ were used to analyse microglial reactivity under regenerative conditions. Degenerative in vivo experiments weren't allowed and the samples to rare to analyse different ages. The study animals were housed at the Translational Animal Research Centre of Johannes-Gutenberg University Mainz, provided with food and water ad libitum. Eyes from marmosets (Callithrix jacchus) aged 3 years $(n=4)$ were obtained from eye cadavers provided by the Institute of Regenerative Medicine, University of Münster, Germany. A 12-hour day-and-night cycle was applied. Meaningful usage of animals' life and reduction of stress and pain during interventions was at highest priority throughout the whole study. Untreated animals served as the controls, respectively.

\section{Induction of experimental glaucoma in vivo}

The IOP was elevated in vivo through cauterization of three episcleral veins, leading to an elevation of the intraocular pressure (IOP). Surgery was performed in female Sprague Dawley rats $(n=5)$ as described by Shareef et al. [38] . Medetomidine hydrochloride (Dorbene vet., Pfizer, USA) and ketamine (Inresa Arzneimittel, Freiburg, Germany) were injected intraperitoneally. Oxybuprocain (Novesine, OmniVision, Germany) was topically applied to the ocular surface. Novaminsulfon (Novalgin, Ratiopharm, Germany) was added to the drinking water to prevent post-operative pain. Animal's general condition and behaviour was observed closely after the intervention and followed daily.
Subsequent elevation of IOP was determined using a Tonolab rodent rebound Tonometer (iCare, Finland) between 9:00 a.m. and 11:00 a.m. on a weekly base for 7 weeks, and a baseline was obtained before surgery. Ten readings of the Tonolab were taken per measurement for each eye and consequently averaged. During IOP measurements, the animals were not anesthetized, and only fixated through handholding to ensure accurate values in terms of IOP and blood pressure. Animals without noticeable IOP elevation or with fluctuating IOP were excluded from the study. Untreated animals served as the corresponding controls $(\mathrm{n}=5)$.

\section{Ex vivo retinal explantation}

Ex vivo experiments were performed with a retinal organ-culture model, described in a variety of different animals $[2,13,31]$. Eyes from healthy female Sprague Dawley rats (each $n=8$ ) aged 3 months and 24 months, were enucleated and transferred into Beta-Isadona (Braun Melsungen, Germany) for a period of five minutes. Subsequently, the eyeball was transferred into a petri-dish containing ice-cold sterile Hank's Balanced Salt Solution (ThermoFisher, USA), which was previously enriched with oxygen. The anterior segment of the eye was taken aside and the intact retina was harvested from the optic cup and flat-mounted on Millipore filters (Millipore; Millicell; Cork, Ireland) with the ganglion cell layer upwards. The vitreous was removed in addition. The retinal explant was transferred into DMEM medium, containing $10 \%$ FCS, $1 \%$ penicillin/streptomycin, 1\% HEPES and incubated for 48 hours at $37^{\circ} \mathrm{C}$ in a $5 \% \mathrm{CO}_{2}$ atmosphere.

\section{High pressure incubation chamber}

A metallic incubation chamber was self-fabricated from steel. The metallic high-pressure incubator fabricated for this purpose with a screw-able cover and an uni-directed valve to allow for entrance of incubator air. The intracameral air-pressure is adjusted with a nanometer with readings in $\mathrm{mmHg}$. Constant air pressure can be obtained over several days. The incubator allowed for increasing the air pressure up to $200 \mathrm{mHg}$ (266,64 hpascal) keeping this pressure stable or changing the pressure on demand. A valve allowed for entrance of $5 \% \mathrm{CO}_{2}$ containing atmosphere from the main incubator (Heraeus, Germany). A manometer was used to continually monitor the air pressure within the high-pressure incubator. Retinal explants described as above were cultured within the high-pressure incubator until they were processed for IHC.

\section{Retinal regeneration experiments}

Optic nerve crush was conducted in female Sprague-Dawley rats $(\mathrm{n}=3 ; 250-300 \mathrm{~g})$, a method previously described by Li et al [22] $(\mathrm{n}=5)$. Briefly, animals were anesthetized as described above, topical drops of $0.4 \%$ oxybuprocaine (Novesine, Novartis) were used for corneal anaesthesia, a lateral canthotomy was performed to the left upper eyelid parallel to the superior orbital edge, fine forceps were used to clamp the exposed optic nerve for 3 seconds, meninges were not clamped so retinal blood flow was not impacted, the incision was sutured and the eye was covered with antibiotic ointment (Floxal, Bausch+Lomb). The right eye was left untreated. Novaminsulfon (Novalgin, Ratiopharm, Germany) was added to the drinking water to reduce any post-operative pain. Animals were sacrificed under $\mathrm{CO}_{2}$ atmosphere 3 days after the operation, retinal explants were prepared as described above. Untreated animals served as the corresponding controls $(n=5)$.

The monkey retinas were prepared as described before [37] ( $\mathrm{n}=4$ each). The rat retinas were prepared accordingly. The animals were 
euthanized and their eyes removed and placed into oxygenated Hank's balanced salt solution (HBSS). After disinfection of the whole eye in $5 \%$ iodide solution for $5 \mathrm{~min}$, they were washed in HBSS for $5 \mathrm{~min}$. All of the following preparation steps were conducted under sterile conditions. After removal of the vitreous body, the retina was isolated and carefully spread on a sterile nitrocellulose membrane (pore size, 45 $\mu \mathrm{m}$; Sartorius, Göttingen, Germany) using fine forceps. The membrane was divided into several tapered pieces $(\varnothing 8)$ and placed with the retinal ganglion cell (RGC) layer on poly-D-lysine- and laminincoated PetriPERM dishes (Sigma-Aldrich, Munich, Germany) (Fig 1a). Serum-free S4 medium (fabricated in the laboratory containing DMEM as basis medium, $1 \%$ penicillin and streptomycin, and $6.5 \mathrm{~g} / \mathrm{l}$ glucose) was added to the retinal culture $(1 \mathrm{ml})$ and incubated for $96 \mathrm{~h}$ in a humidified atmosphere containing $5 \% \mathrm{CO}_{2}$. The number of outgrowing axons was determined after 24,48 , and $72 \mathrm{~h}$ in culture using an inverted phase-contrast microscope ( $\times 200$ magnification; Axiovert 135, Carl Zeiss Jena, Germany) or time-lapse video microscopy (Axiovert 35, Carl Zeiss Jena). In the control condition, retinal samples were placed without a filter in the media, cultured for 3 days without outgrowing axons for the monkeys or without previous ONC regarding rats.

\section{Immunohistochemistry of retinal explants and cross sections}

Visualization of microglial cells was conducted using a rabbit antibody against the ionized calcium binding adaptor molecule (anti Iba-1, Wako, Germany) in retinal flatmounts for the ex vivo- and in retinal cross-section staining's for the in vivo experiments. In brief, retinal explants were fixed in formalin-solution ( $4 \%$ para-formaldehyde in Phosphate-buffered saline, $\mathrm{pH} 7.4$ ), transferred in $30 \%$ sucrose solution overnight and finally frozen in methylbutan for 10 seconds (Merck, Germany). The cross-section tissue was blocked for one hour with $1 \%$ bovine serum albumin, $0.5 \%$ tween, $0.5 \%$ triton- $\mathrm{x}$ and $0.5 \%$ normal goat serum in PBS. Both explants and cross section tissues were incubated with the primary anti Iba- 1 antibody overnight at $4^{\circ} \mathrm{C}$. After washing, the goat anti-rabbit Cy3 secondary antibody (Linaris, Germany) was incubated for two hours at room temperature. Immunofluorescent microglia were further visualized with a fluorescent microscope (Axiophot Carl Zeiss, Germany).

\section{Grading and quantification of retinal microglia cells}

Iba-1 positive microglial cells were graded into two different morphological phenotypes: ramified and branched like microglia were regarded as rested, non-activated cells. Roundish Iba-1 positive cells with fewer branches were considered activated. A cell-body diameter of $10 \mu \mathrm{m}$ was set as the cut-off criterion. Microglia with a cell body larger than $10 \mu \mathrm{m}$ with thick and hardly any branches were regarded as activated. In contrast, microglia possessing smaller cell bodies with long, lean and relatively more branches were considered to be nonactivated cells. Macrophages, which are also known to express Iba-1, were distinguished by their much larger size and lack of branches. For retinal flat mounts, pictures were taken in the same eight locations of each quadrant of the retina, and for retinal cross-sections, three unrelated cross-sections of each retina were examined, pictures were taken from 4 locations of each cross-section, stained microglia were graded and quantified, in retinal cross-sections, stained microglia were also categorized into different retinal layers (Figure 1).

\section{Statistical analysis}

Data was analyzed using Prism 8 software (GraphPad Software, Inc., San Diego, CA, USA). Significance of difference between groups was determined by t-test and one-way ANOVA, $\mathrm{p}<0.05$ was considered to be statistically significant. The averaged cell number was calculated per $\mathrm{mm}^{2} \pm$ standard deviation. All data values are expressed as the mean \pm SD or mean \pm SEM, either of which is indicated in the Figure.

\section{Results}

\section{Microglial quantification and grading after elevated IOP in vivo}

Episcleral vein occlusion resulted in a significant elevation of IOP and a significant RGC loss as shown in our previous studies [1]. Elevated IOP was maintained for seven weeks and rats were then harvested to analyse any possible microglial changes due to elevated pressure in vivo.

Elevated IOP in vivo did not lead to an increase in the total amount of microglial cells within the retina (Figure 2A). However, the proportion of activated and resting microglia changed dramatically (Figure 2B). In the control $20.6 \%$ of microglia $\left(2.14 \pm 0.13 / \mathrm{mm}^{2}\right)$ were activated with the number of resting microglia four times that high $\left(8.57 \pm 0.86 / \mathrm{mm}^{2}, \mathrm{p}<0.001\right)$. IOP elevation in vivo resulted in $40 \%$ of activated microglia $\left(4.35 \pm 1.24 / \mathrm{mm}^{2}\right)$ which was significantly higher than in the comparative controls $(\mathrm{p}<0.01)$. Thus, IOP elevation in vivo led to a considerable shift towards an activation of microglia cells.
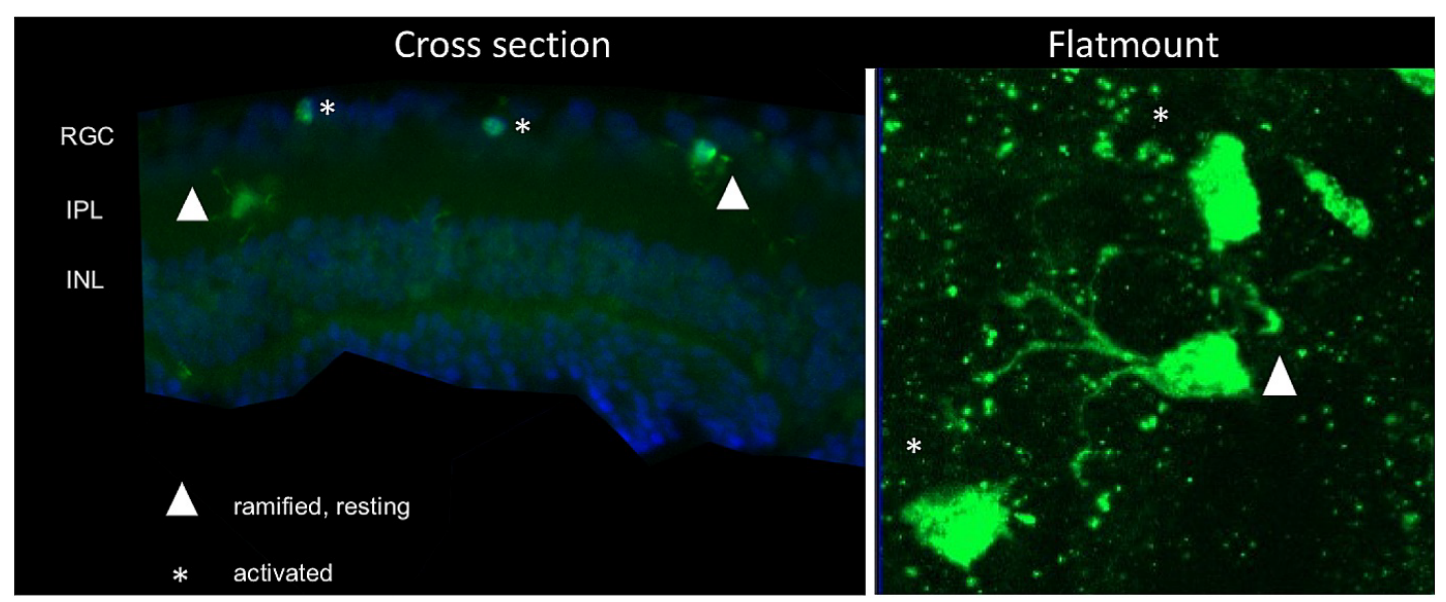

Figure 1. Overview of the microglial grading system in retinal cross-sections and retinal flat mounts. Microglia with larger cell bodies and very few and thick branches were considered as activated microglia (marked with a star). Cells (marked with triangle) with smaller cell bodies and numerous long lean branches, were considered as ramified, non-activated microglia 
A

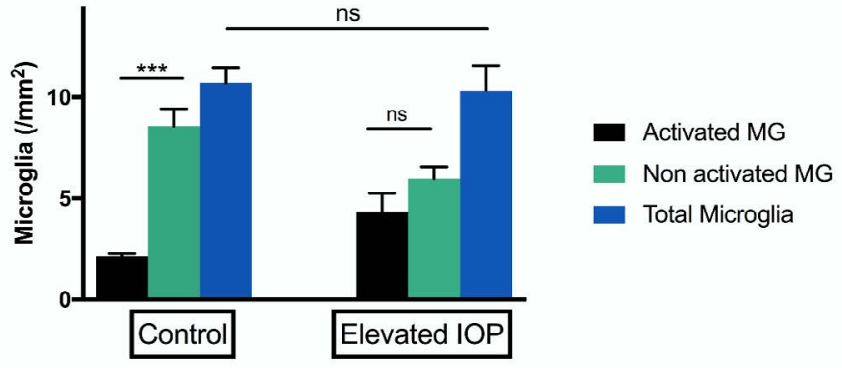

B

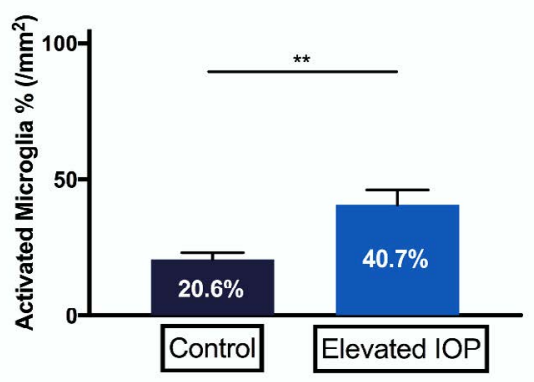

Figure 2. Grading and quantification of microglial cells in experimental glaucoma in rats in vivo. (A) Iba-1 stained retinal cross-sections from control eyes showed significantly more resting microglia than activated ones $(* * * \mathrm{p}<0.001, \mathrm{n}=5, \pm \mathrm{SEM})$. Eyes, which were exposed to elevated IOP for a period of seven weeks showed no significant difference between activated and resting microglia. (B) In control eyes, there were $20.6 \pm 2.395 \%$ activated microglial cells. Eyes exposed to elevated IOP showed a significantly higher percentage of activation ( $40.7 \pm 5.237 \%$ cells) $(* * \mathrm{p}<0.01, \mathrm{n}=5$, mean $\pm \mathrm{SEM})$

\section{Microglial changes due to ageing ex vivo and stressors}

To investigate the impact of aging on microglial changes we used retinal tissue from female Sprague Dawley rats of different ages ( 3 and 24 months; each $n=8)$ ). There was a significant increase in the total number of microglial cells in older rats (24 month, $118.4 \pm 5.29 / \mathrm{mm}^{2}$, compared to younger rats ( 3 month, $48 \pm 4.411 / \mathrm{mm}^{2}$, $\left.\mathrm{p}<0.0001\right)$. Besides that, there was a significant rise in activated microglia in the older animals (24 month: $41.07 \pm 2.397 / \mathrm{mm}^{2}$; 3 month: $11.52 \pm 2.968 / \mathrm{mm}^{2}$, $\mathrm{p}<0.0001$ ), whilst the proportion of activated microglia in young and old animals did not significantly differ from each other.

In addition to that, retinal explants of differently aged animals were cultured over 48 hours with or without additional external pressure of $40 \mathrm{mmHg}$ (each $\mathrm{n}=8$ ). Additional pressure in vitro did not change (Figure 3) the absolute number of total microglia in the retina as to expect.

However, under pressure of $40 \mathrm{mmHg}$ over 48 hours, the flatmounts from younger animals showed $42.23 \%$ activated microglial cells $(p<0.05)$, whereas in older animals, only $19.6 \%$ of activated microglia were observed, which was significantly less $(\mathrm{p}<0.001)$.

\section{Microglial changes under regenerative conditions}

Next, we investigated the reaction of microglia under regenerative conditions. After cultivation under regenerative conditions for 5 days, outgrowing neurites were observed and subsequently photographed under a binocular microscope with a 40-fold magnification (Figure 4).

Retinal explants were stained against Iba- 1 antibody after 48 hours of cultivation. Compared to controls, which were not subjected to optic nerve crush prior to cultivation, cultivation under regenerative conditions significantly increased the number of activated microglial cells ( $\mathrm{p}<0.0001$ ). $58.55 \%$ of total microglial cells were activated under regenerative conditions, and only $41 \%$ in controls (Figure 5).

\section{Microglial changes under regenerative conditions in primates ex vivo}

We furthermore investigated microglial activation under regenerative conditions in callithrix, RGC axonal regeneration was proved in our previous study [28], subject to $48 \mathrm{~h}$ cultivation under regenerative conditions, there was no noticeable microglial activation. Consequently, when compared to the activation pattern in rats, there was significantly less net microglial activation, in fact, there was even less activated microglia in marmosets subjected to $48 \mathrm{~h}$ cultivation under regenerative conditions (Figure 6).

\section{Discussion}

The microglia are the immune cells of the CNS and therewith the retina [21]. In healthy retinas they perform tissue surveillance tasks, in presence of disease or injury, microglia protect neural tissue and can play a key role in the defense and repair of damaged tissue, because they are rapidly activated in response to a wide variety of neural injuries [18]. However, their response to injury can result in uncontrolled immune-phenotypic and morphological changes putting neuronal survival at risk due to excessive inflammation. This is considered one of the major factors of aged-related neurodegenerative diseases of the CNS such as glaucoma. Purpose of this study was to analyze 1. Agedependent differences in microglial responses; 2 . Exploring the role in degeneration and regeneration and; 3 . Looking into differences between rodents and mammalians. 

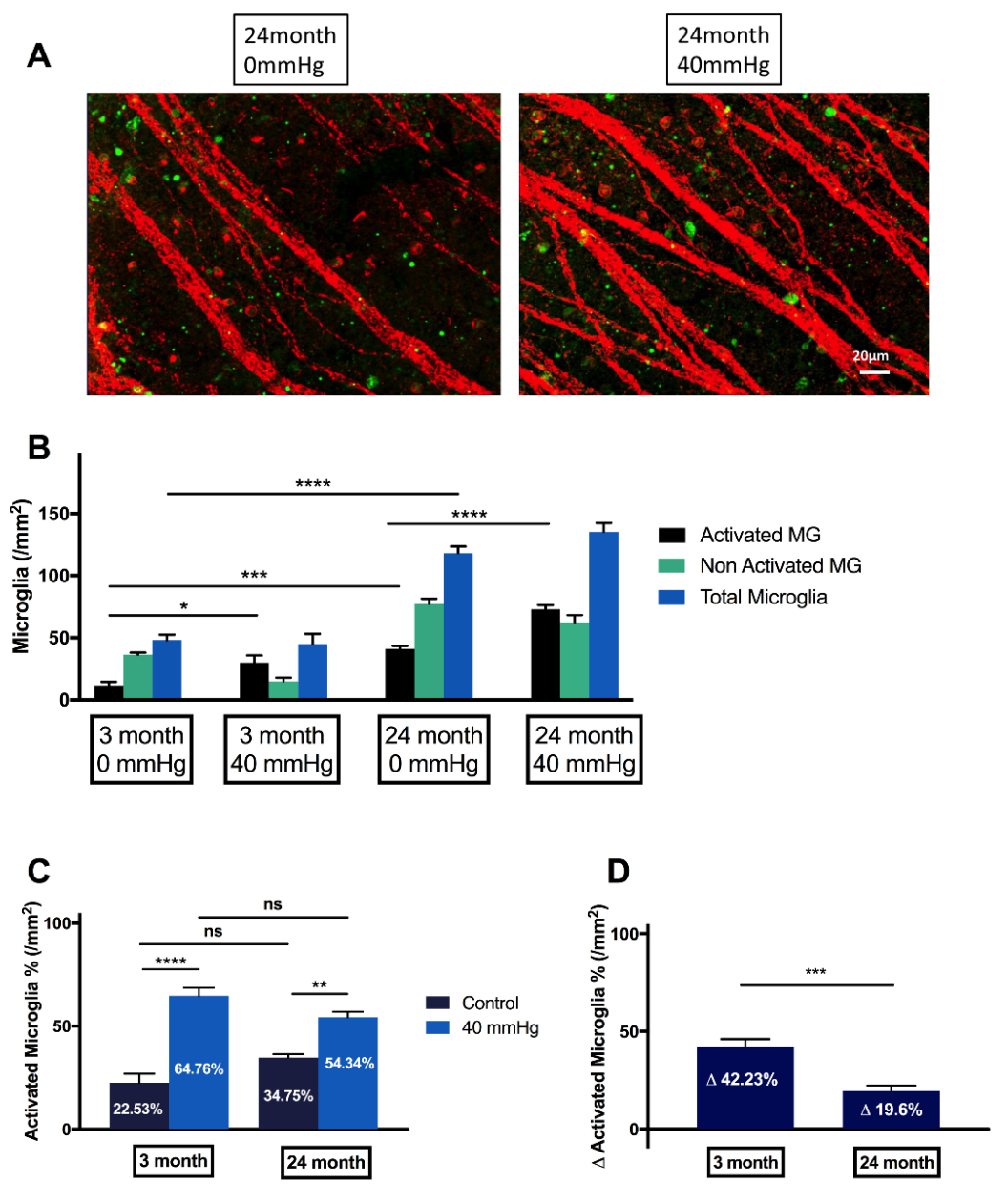

Figure 3. Grading and quantification of microglial cells in experimental glaucoma in rats ex vivo. (A) Older animals possess significantly more activated microglial cells than juvenile animals. (B) Additional pressure doesn 't have an impact on total cell numbers (C) However, additional external pressure led to a significantly higher percentage of activated microglial cells in juvenile animals $(42.23 \pm 3.896 \%)$ compared to elderly animals $(19.6 \pm 2.704 \%) .\left({ }^{*} \mathrm{p}<0.05,{ }^{* * *} \mathrm{p}<0.001,{ }^{*} * * * \mathrm{p}<0.0001, \mathrm{n}=8\right.$, mean $\left.\pm \mathrm{SEM}\right)$

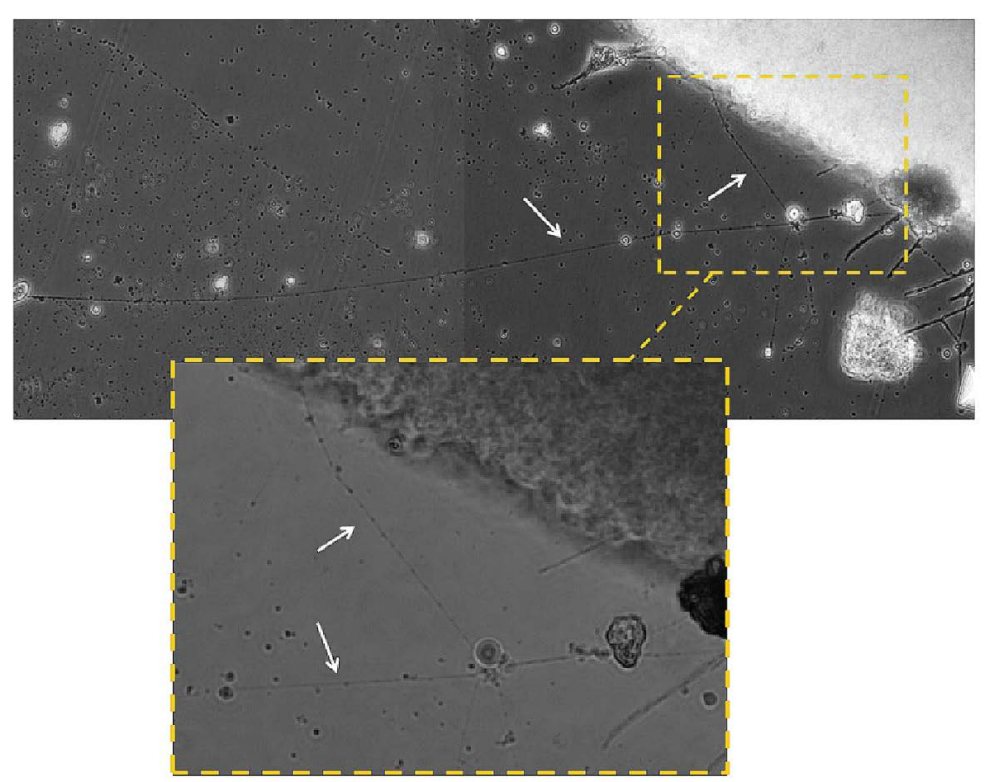

Figure 4. After cultivation under regenerative conditions for 5 days, outgrowing neurites were observed and subsequently photographed under a binocular microscope with a 40 -fold magnification 
A
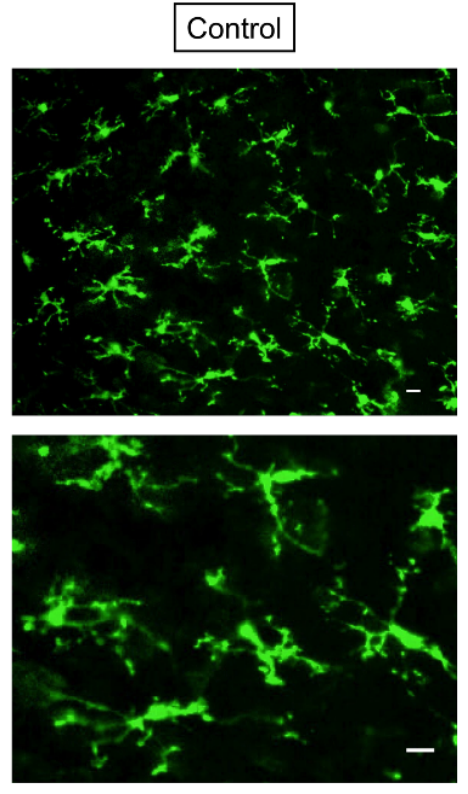

B

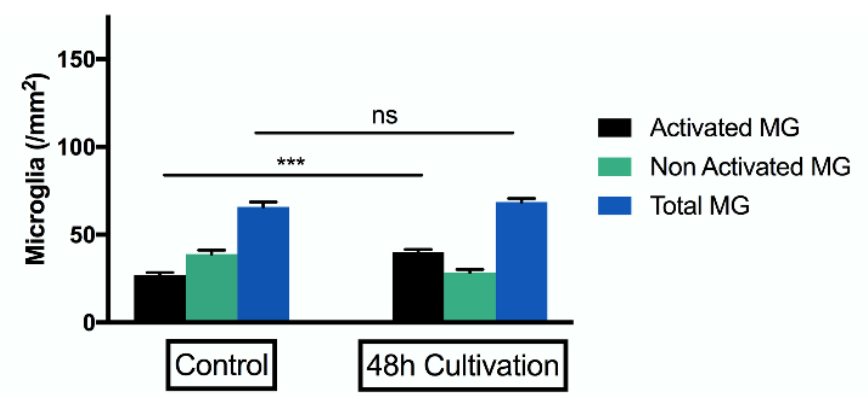

C

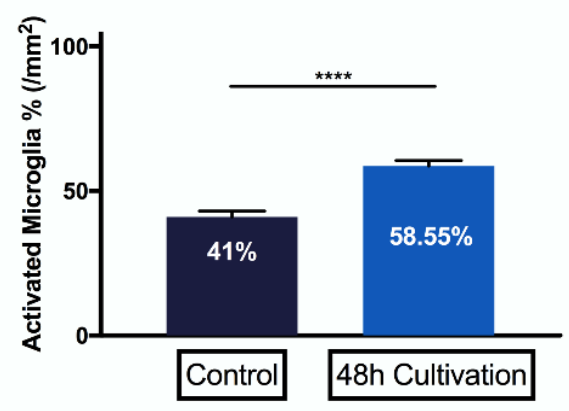

D

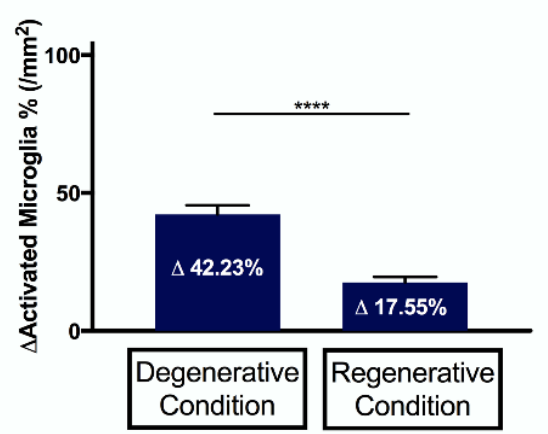

Figure 5. Grading and quantification of microglial cells under regenerative conditions. (A) representative fluorescence microscopy of Iba- 1 staining of retinal explants of rats, 48 hours after culturing under regenerative conditions. (B-C) There is no significant difference in total number of microglial cells under regenerative conditions. Iba-1 stained retinal explants from control eyes showed $41 \pm 1.995 \%$ activated microglial cells in regenerative conditions. Eyes, which underwent optic nerve crush prior to culture, showed significantly more activation ( $58.55 \pm 5.225 \%$ ) of microglial cells $(* * * * \mathrm{p}<0.0001, \mathrm{n}=3$, mean \pm SEM). (D) Comparison of grading and quantification of microglia cells in degeneration and regeneration. Due to the degenerative condition, $42.23 \pm 3.293 \%$ more microglia cells are activated, whereas under regenerative conditions, $17.55 \pm 2.048 \%$ more microglial cells were activated. There were significantly more dramatic changes in activation of microglial cells in degeneration. $(* * * * \mathrm{p}<0.0001, \mathrm{n}=6$, mean $\pm \mathrm{SEM})$ 

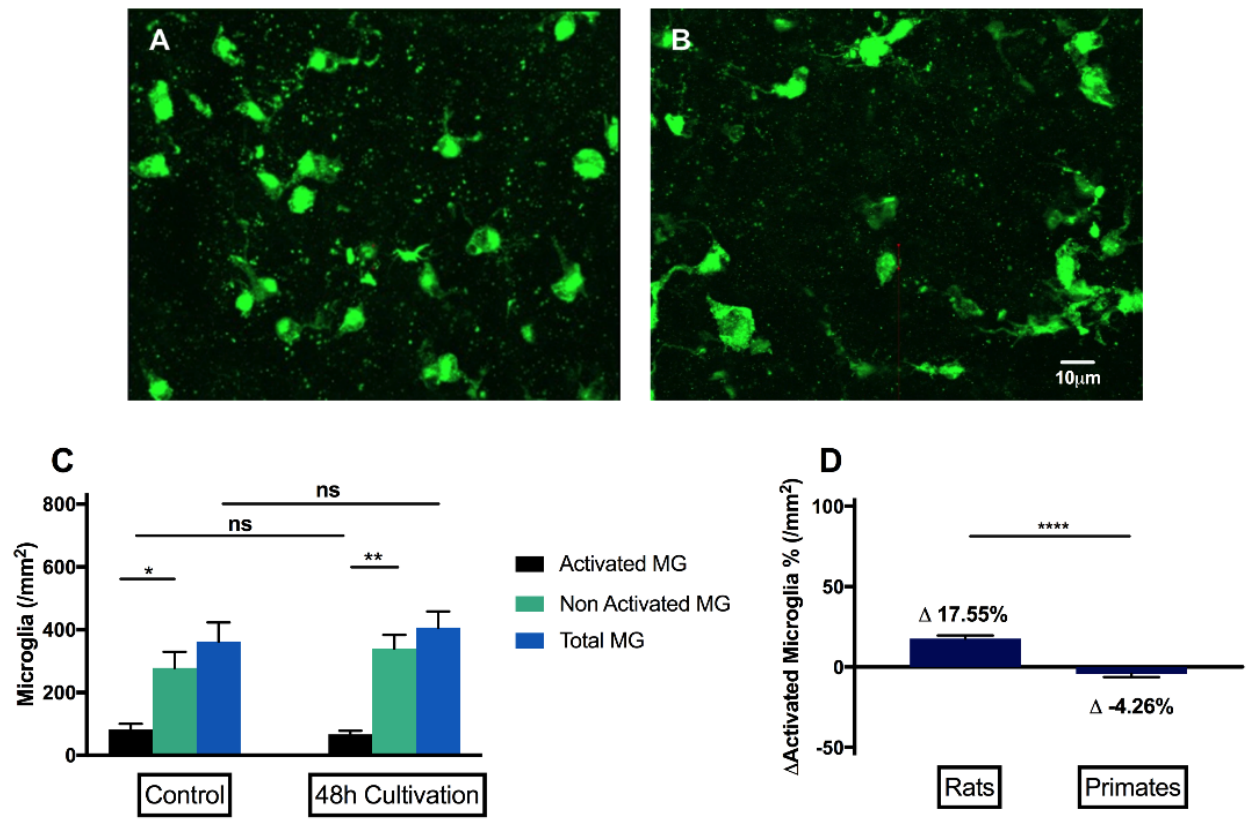

Figure 6. Grading and quantification of microglial cells under regeneration in primates ex vivo. (A) representative fluorescence microscopy of Iba-1 staining of retinal explants of primates, 48 hours after culturing under regenerative conditions. (B)In reaction to the regenerative condition, there is no significant difference in the number of activated microglia and total microglia. (C)Subject to the same ex vivo regenerative condition, $48 \mathrm{~h}$ under cultivation, there are $17.55 \%$ activated microglia in the rat, whereas $4.26 \%$ less activated microglia in primates. $(* * * \mathrm{p}<0.0001, \mathrm{n}=6$, mean \pm SEM $)$

Microglial activation is the principal component of neuroinflammation, which provides the first line of defense when neural homeostasis is disrupt [7]. In this study, we had the following findings: 1 . both in vivo and ex vivo degenerative conditions resulted in a significant rise of activated microglia, whereas the total number of microglia remained unchanged. 2. With ageing the total number of microglia increases significantly as well as the number of activated microglia. The proportion of activated microglia cells towards total microglia is not significantly different between the different age groups. However, as a response to elevated pressure, there's significantly less activation of microglia cells in old animals compared to younger ones.3. Under regenerative conditions we found a significant increase in activated microglial cells. However, compared to degenerative conditions, there was significantly less activation of microglia cells. 4 . In primates, the activation pattern of microglial cells under regenerative conditions is different from rats.

As the housekeeping immune cell of retina, microglia are the first responders to infections or injuries[14], and generate inflammatory responses to regulate retinal homeostasis, by releasing interleukin$1 \beta$ (IL-1 $\beta$ ), IL-12, TNF- $\alpha$ and nitric oxide synthase (iNOS) $[8,42]$. Fundamentally, inflammation is an immune response to protect the neurons against harm, normally once the damage is taken care of, neuroinflammation is also downregulated for proper healing. Activated microglia on one hand contribute to phagocytic clearance of dying or malfunctioning neurons, by releasing neurotrophic and anti-inflammatory molecules [43], and furthermore to maintain and support neuronal survival and neurogenesis $[11,24,45]$, but on the other hand, over-activated microglia can contribute to disease progression by releasing pro-inflammatory factors, reactive oxygen species (ROS) and tumor necrosis factor- $\alpha,[12]$ which are neurotoxic and interleukins such as IL-6, which expressed by microglia, exert both anti- and proinflammatory effects [44].
We observed a significant rise of activated microglia under both in vivo and ex vivo degenerative conditions. Studies showed, that early in the glaucomatous process, microglia produce TGF- $\beta 2$ (Transforming growth factor $\beta 2$ ), which may downregulate some of the neurodestructive pathways, and Matrix metalloproteinases (MMPs), which may degrade the extracellular matrix (ECM). Also, microglia phagocytose cellular debris, apparently a byproduct of the neurodegeneration in the optic nerve head $(\mathrm{ONH})$. Late in the glaucomatous process, when severe optic nerve degeneration is occurring, microglia may contribute detrimentally to the neurodestruction by producing TNF-a and NOS-2.

It has known, that, microglia has a pathogenic role in degenerative CNS diseases such as Alzheimer's dementia (AD) and Parkinson's disease, characterized by selective loss of neurons in distinct regions of the brain. And on site of neurodegeneration, microglia are activated despite a clear sign of inflammatory response [39].

Microglia are active housekeepers and function to promote neuronal growth and activity, however, with advanced age, increased oxidative stress, dysregulated inflammatory signaling and cellular senescence, defects in phagocytosis impair their ability to perform the most essential of homeostatic functions, including immune surveillance and debris clearance.

One of the major risk factors in glaucoma is age. The uncontrolled immune-phenotypic and morphological changes of microglia cells putting neuronal survival at risk due to excessive inflammation is considered one of the major factors of aged-related neurodegenerative diseases of the CNS such as glaucoma. Age-related neurodegeneration diseases like glaucoma share similar pathogenesis, such as microglialmediated inflammations in neuronal cells, activated microglia can release pro-inflammatory cytokines which can aggravate and propagate neuroinflammation, thereby degenerating neurons and impairing brain as well as retinal function [14]. Microglial activation is one of the 
signs of aging [17]. In our study, elderly animals possessed $50 \%$ more activated microglia than juvenile animals, which might explain the agedependency of the disease.

There are abundant studies focusing on the effects of aging on macrophages and other lymphoid-associated myeloid cells, but understanding of microglial function changing in aging is much less clear. Normal aging has significant effects on endocytic pathways, including the phagocytic uptake of debris [32]. This impairment in function potentially leads to amyloid $\beta$ accumulating and further formation in extracellular environment, and subsequently leads to further activation of microglia $[5,36]$.

We observed, that microglia in juvenile animals are much more sensitive to the change of the environment. There are almost $42.23 \%$ more activated microglia in reaction to increased IOP, whilst in older animals, only $18.6 \%$ more activated microglia, which is significantly less activation. At the functional level, microglia already demonstrated an age-related decrease in phagocytic behavior [30]. The limited microglial activation in older animals makes them more vulnerable to additional assault and less competent in executing their housekeeper functions.

The development of novel medicaments that capable of directly modulating phagocytic activity of microglia is a realistic aim.

The faith of glaucoma is irreversible retinal ganglion cell loss (RGC) and the disability to regenerate new axons. While the role of microglia in a degenerative neuronal state, as in glaucoma, was investigated deeply in recent years, their differentiated function in neuronal regeneration is rather unknown until today. Questions remain, if microglial activation under different stimuli promotes or compromises neuronal survival. Upon the completion of development, neuron plasticity decreases dramatically to close to zero, as first noted by Santiago Ramón y Caj, et al. [2]. It is presumed that this failure is attributable to two main elements: (1) the down-regulation of growth-related genes and proteins resulting in a poor intrinsic ability for neurons to regenerate and (2) the expression of growth-inhibiting proteins by glial cells and/or other components of the CNS. The microglial cells are supposed to play a major role in this context by expressing neuro-inhibitory substances. We found under regenerative conditions a significant increase in activated microglia cells. However, compared to degenerative conditions, there was significantly less activation of microglia cells. Consistent with in vivo experiments, supplying NTFs such as BDNF and CNTF, microgliasuppressing factors, and NT-4/5 to retinal explants promotes short-term RGC survival and axon regeneration in vitro. Thus, microglia inhibit regeneration. Especially in degenerative conditions where microglia is overactivated, regeneration might become problematic.

Loads of glaucoma models don't reflect the situation in humans and there is a lack of good models. One problem is the species. Most studies are done in rodents, but primates might react differently. Our study showed that microglia response in monkeys under regenerative conditions was completely different from responses in rats. This has to be beard in mind when translating from lab to clinic or animal models to humans. Rodents have a very different immune system.

\section{Conclusions}

In conclusion this is to best of our knowledge the first study comparing microglia activation under regenerative and regenerative conditions, different age groups and different species. Our findings however showed that microglia and their activation play an important role throughout ageing, degeneration and regeneration and differs in different species. Thus tackling microglia might be a clue to help in agedependant diseases of the CNS such as glaucoma.

\section{Funding}

This work was supported by the Deutsche Forschungsgemeinschaft under Grant PR1569-1-1 and Th386-20-1.

\section{Declaration of interest}

None.

\section{References}

1. Anders, F.; Liu, A.; Mann, C.; Teister, J.; Lauzi, J., et al., (2017) The Small Heat Shock Protein alpha-Crystallin B Shows Neuroprotective Properties in a Glaucoma Animal Model. Int J Mol Sci, 18.

2. Bell, K.; Wilding, C.; Funke, S.; Perumal, N.; Beck, S., et al., (2016) Neuroprotective effects of antibodies on retinal ganglion cells in an adolescent retina organ culture. $J$ Neurochem, 139: 256-269.

3. Bosco, A.; Steele, M. R.; Vetter, M. L., (2011) Early microglia activation in a mouse model of chronic glaucoma. J Comp Neurol, 519: 599-620.

4. Bosco, A.; Inman, D. M.; Steele, M. R.; Wu, G.; Soto, I., et al., (2008) Reduced retina microglial activation and improved optic nerve integrity with minocycline treatment in the DBA/2J mouse model of glaucoma. Invest Ophthalmol Vis Sci, 49: 1437-1446.

5. Floden, A. M.; Combs, C. K., (2011) Microglia demonstrate age-dependent interaction with amyloid-beta fibrils. J Alzheimers Dis, 25: 279-293.

6. Frank-Cannon, T. C.; Alto, L. T.; McAlpine, F. E.; Tansey, M. G., (2009) Does neuroinflammation fan the flame in neurodegenerative diseases? Mol Neurodegener, 4: 47 .

7. Glass, C. K.; Saijo, K.; Winner, B.; Marchetto, M. C.; Gage, F. H., (2010) Mechanisms underlying inflammation in neurodegeneration. Cell, 140: 918-934.

8. Gonzalez, H.; Elgueta, D.; Montoya, A.; Pacheco, R., (2014) Neuroimmune regulation of microglial activity involved in neuroinflammation and neurodegenerative diseases. J Neuroimmunol, 274: 1-13.

9. Guadagno, J.; Swan, P.; Shaikh, R.; Cregan, S. P., (2015) Microglia-derived IL-1beta triggers p53-mediated cell cycle arrest and apoptosis in neural precursor cells. Cell Death Dis, 6: e1779.

10. Guadagno, J.; Xu, X.; Karajgikar, M.; Brown, A.; Cregan, S. P., (2013) Microgliaderived TNFalpha induces apoptosis in neural precursor cells via transcriptional activation of the Bcl-2 family member Puma. Cell Death Dis, 4: e538.

11. Harry, G. J.; McPherson, C. A.; Wine, R. N.; Atkinson, K.; Lefebvre d'Hellencourt, C., (2004) Trimethyltin-induced neurogenesis in the murine hippocampus. Neurotox Res, 5: 623-627.

12. Huang, H. Y.; Chang, H. F.; Tsai, M. J.; Chen, J. S.; Wang, M. J., (2016) 6-Mercaptopurine attenuates tumor necrosis factor-alpha production in microglia through Nur77-mediated transrepression and PI3K/Akt/mTOR signaling-mediated translational regulation. J Neuroinflammation, 13: 78

13. Johnson, T. V.; Martin, K. R., (2008) Development and characterization of an adult retinal explant organotypic tissue culture system as an in vitro intraocular stem cell transplantation model. Invest Ophthalmol Vis Sci, 49: 3503-3512.

14. Jones, E. V.; Bouvier, D. S., (2014) Astrocyte-secreted matricellular proteins in CNS remodelling during development and disease. Neural Plast, 2014: 321209.

15. Kettenmann, H.; Hanisch, U. K.; Noda, M.; Verkhratsky, A., (2011) Physiology of microglia. Physiol Rev, 91: 461-553.

16. Kim, C. C.; Nakamura, M. C.; Hsieh, C. L., (2016) Brain trauma elicits non-canonical macrophage activation states. J Neuroinflammation, 13: 117

17. Koellhoffer, E. C.; McCullough, L. D.; Ritzel, R. M., (2017) Old Maids: Aging and Its Impact on Microglia Function. Int J Mol Sci, 18.

18. Kreutzberg, G. W., (1996) Microglia: a sensor for pathological events in the CNS Trends Neurosci, 19: 312-318.

19. Lee, J. E.; Liang, K. J.; Fariss, R. N.; Wong, W. T., (2008) Ex vivo dynamic imaging of retinal microglia using time-lapse confocal microscopy. Invest Ophthalmol Vis Sci, 49: 4169-4176.

20. Levkovitch-Verbin, H.; Kalev-Landoy, M.; Habot-Wilner, Z.; Melamed, S., (2006) Minocycline delays death of retinal ganglion cells in experimental glaucoma and after optic nerve transection. Arch Ophthalmol, 124: 520-526. 
21. Li, L.; Eter, N.; Heiduschka, P., (2015) The microglia in healthy and diseased retina. Exp Eye Res, 136: 116-130.

22. Li, Y.; Schlamp, C. L.; Nickells, R. W., (1999) Experimental induction of retinal ganglion cell death in adult mice. Invest Ophthalmol Vis Sci, 40: 1004-1008.

23. Liu, B.; Wang, K.; Gao, H. M.; Mandavilli, B.; Wang, J. Y., et al., (2001) Molecular consequences of activated microglia in the brain: overactivation induces apoptosis. $J$ Neurochem, 77: 182-189.

24. Liu, B.; Gao, H. M.; Wang, J. Y.; Jeohn, G. H.; Cooper, C. L., et al., (2002) Role of nitric oxide in inflammation-mediated neurodegeneration. Ann N Y Acad Sci, 962: 318-331.

25. London, A.; Benhar, I.; Schwartz, M., (2013) The retina as a window to the brain-from eye research to CNS disorders. Nat Rev Neurol, 9: 44-53.

26. Magni, P.; Ruscica, M.; Dozio, E.; Rizzi, E.; Beretta, G., et al., (2012) Parthenolide inhibits the LPS-induced secretion of IL-6 and TNF-alpha and NF-kappaB nuclear translocation in BV-2 microglia. Phytother Res, 26: 1405-1409.

27. Menzies, F. M.; Henriquez, F. L.; Alexander, J.; Roberts, C. W., (2010) Sequential expression of macrophage anti-microbial/inflammatory and wound healing markers following innate, alternative and classical activation. Clin Exp Immunol, 160: 369-379.

28. Mertsch, S.; Schlicht, K.; Melkonyan, H.; Schlatt, S.; Thanos, S., (2018) snRPN controls the ability of neurons to regenerate axons. Restor Neurol Neurosci, 36: 31-43.

29. Nayak, D.; Roth, T. L.; McGavern, D. B., (2014) Microglia development and function. Annu Rev Immunol, 32: 367-402.

30. Njie, E. G.; Boelen, E.; Stassen, F. R.; Steinbusch, H. W.; Borchelt, D. R., et al., (2012) Ex vivo cultures of microglia from young and aged rodent brain reveal age-related changes in microglial function. Neurobiol Aging, 33: 195 e191-112.

31. Ogilvie, J. M.; Speck, J. D.; Lett, J. M.; Fleming, T. T., (1999) A reliable method for organ culture of neonatal mouse retina with long-term survival. J Neurosci Methods, 87: 57-65.

32. Orre, M.; Kamphuis, W.; Osborn, L. M.; Jansen, A. H. P.; Kooijman, L., et al., (2014) Isolation of glia from Alzheimer's mice reveals inflammation and dysfunction. Neurobiol Aging, 35: 2746-2760.

33. Ramirez, A. I.; de Hoz, R.; Salobrar-Garcia, E.; Salazar, J. J.; Rojas, B., et al., (2017) The Role of Microglia in Retinal Neurodegeneration: Alzheimer's Disease, Parkinson, and Glaucoma. Front Aging Neurosci, 9: 214.
34. Ransohoff, R. M., (2016) A polarizing question: do M1 and M2 microglia exist? Nat Neurosci, 19: 987-991.

35. Ransohoff, R. M.; Cardona, A. E., (2010) The myeloid cells of the central nervous system parenchyma. Nature, 468: 253-262.

36. Ritzel, R. M.; Patel, A. R.; Pan, S.; Crapser, J.; Hammond, M., et al., (2015) Age- and location-related changes in microglial function. Neurobiol Aging, 36: 2153-2163.

37. Rose, K.; Schroer, U.; Volk, G. F.; Schlatt, S.; Konig, S., et al., (2008) Axonal regeneration in the organotypically cultured monkey retina: biological aspects, dependence on substrates and age-related proteomic profiling. Restor Neurol Neurosci, 26: 249-266.

38. Shareef, S. R.; Garcia-Valenzuela, E.; Salierno, A.; Walsh, J.; Sharma, S. C., (1995) Chronic ocular hypertension following episcleral venous occlusion in rats. Exp Eye Res, 61: 379-382.

39. Stoll, G.; Jander, S., (1999) The role of microglia and macrophages in the pathophysiology of the CNS. Prog Neurobiol, 58: 233-247.

40. Streit, W. J.; Conde, J. R.; Fendrick, S. E.; Flanary, B. E.; Mariani, C. L., (2005) Role of microglia in the central nervous system's immune response. Neurol Res, 27: 685-691.

41. Tambuyzer, B. R.; Ponsaerts, P.; Nouwen, E. J., (2009) Microglia: gatekeepers of central nervous system immunology. J Leukoc Biol, 85: 352-370.

42. Varnum, M. M.; Ikezu, T., (2012) The classification of microglial activation phenotypes on neurodegeneration and regeneration in Alzheimer's disease brain. Arch Immunol Ther Exp (Warsz), 60: 251-266.

43. Walton, N. M.; Sutter, B. M.; Laywell, E. D.; Levkoff, L. H.; Kearns, S. M., et al., (2006) Microglia instruct subventricular zone neurogenesis. Glia, 54: 815-825.

44. Wang, B.; Chen, T.; Wang, J.; Jia, Y.; Ren, H., et al., (2018) Methamphetamine modulates the production of interleukin- 6 and tumor necrosis factor-alpha via the cAMP/PKA/CREB signaling pathway in lipopolysaccharide-activated microglia. Int Immunopharmacol, 56: 168-178.

45. Ziv, Y.; Ron, N.; Butovsky, O.; Landa, G.; Sudai, E., et al., (2006) Immune cells contribute to the maintenance of neurogenesis and spatial learning abilities in adulthood. Nat Neurosci, 9: 268-275.

Copyright: (C2021 Liu H. This is an open-access article distributed under the terms of the Creative Commons Attribution License, which permits unrestricted use, distribution, and reproduction in any medium, provided the original author and source are credited. 